\title{
Applying EVM Principles to Tevatron Beam Position Monitor Project
}

\author{
Bakul Banerjee \\ Fermi National Accelerator Laboratory \\ POB 500 \\ Batavia, IL 60510
}

\begin{abstract}
At Fermi National Accelerator Laboratory (Fermilab), the Tevatron high energy particle collider must meet the increasing scientific demand of higher beam luminosity. To achieve this higher luminosity goal, U. S. Department of Energy (DOE) sponsored a major upgrade of capabilities of Fermilab's accelerator complex that spans five years and costs over fifty million dollars. Tevatron Beam Position Monitor (BPM) system upgrade is a part of this project, generally called RunII upgrade project. Since the purpose of the Tevatron collider is to detect the smashing of proton and anti-protons orbiting the circular accelerator in opposite directions, capability to detect positions of both protons and antiprotons at a high resolution level is a desirable functionality of the monitoring system. The original system was installed during early $1980 \mathrm{~s}$, along with the original construction of the Tevatron. However, electronic technology available in 1980 s did not allow for the detection of significantly smaller resolution of antiprotons.
\end{abstract}

The objective of the upgrade project is to replace the existing BPM system with a new system utilizing capabilities of modern electronics enhanced by a front-end software driven by a real-time operating software. The new BPM system is designed to detect both protons and antiprotons with increased resolution of up to an order of magnitude. The new system is capable of maintaining a very high-level of data integrity and system reliability. The system consists of $27 \mathrm{VME}$ crates installed at 27 service buildings around the Tevatron ring servicing 236 beam position monitors placed underground, inside the accelerator tunnel. Each crate consists of a single Timing Generator Fanout module, custom made by Fermilab staff, one MVME processor card running VxWorks 5.5, multiple Echotek Digital Receiver boards complimented by custom made Filter Board. The VxWorks based front-end software communicates with the Main Accelerator Control software via a special-purpose network called ACNET. New software algorithms are designed to decouple antiproton signals from the mixed proton and antiproton signals.

Along with many technical challenges, it was necessary to meet various project management challenges as well. Since 1999, U.S. Department of Energy (DOE) has taken various measures to improve project management of the acquisition of capital assets. U.S. National Laboratories, with their charter of driving scientific efforts of DOE, have put into place project management methodologies recommended by DOE. One of the outcomes of such measures is the deployment of a formal project management system for large projects. This recommendation is detailed in the DOE order M 413.3. The Tevatron BPM Upgrade project, as a part of the Run II Upgrade project, used the methodology prescribed in the DOE order. Particular attention was concentrated on the performance management since the accelerator complex will be in operation for several years in the future. Because of the cost and schedule constraints on the project, it was also important to deploy Earned Value Management (EVM) methodology recommended by the DOE orders.
In this paper, we discuss how the earned value project management methodology was implemented using a work breakdown structure based on system life cycle phases. Tevatron BPM upgrade project, now nearing completion, has been a significant learning experience for the organization. We discuss important lessons learned from the phase-dependant earned value management project model. Hardware and software co-design along with simultaneously developed high-precision algorithm was a major challenge. Since formal project management process is not fully ingrained into the cultural environment of the laboratory dedicated to high-energy physics research, we also gained significant experience with the planning for schedule and cost in this environment. We also present a set of earned value project management data that may serve as a baseline for future projects.

\section{INTRODUCTION}

Over last thirty years, international scientific communities continue to demand larger scientific facilities to conduct cutting edge research. To meet this need, U.S. National Laboratories, sponsored by US Department of Energy (DOE), built several large user facilities, one of the popular items being particle accelerators. Scientific innovations form the basis of these projects. However, it requires talents of engineers and project managers to translate the concept into a real facility. As aptly stated in the Report of the Project Management Panel of the University of California, President's Council on the National Laboratories, "It is recognized that informality and lack of structure are often conductive to scientific innovations and creativity and may be the lifeblood for the type of scientific talent that is responsible for creating a unique, cutting edge facility. However, once a facility/project proposal has been selected for implementation, a transition in thinking must occur in order to translate those ideas into the reality of a working facility while adhering to constraints in budget and time that are expected by the sponsor." [1] This concept of organization is further enhanced by various directives on performance based management. [2] The guidance on OMB300 on implementing Earned Value Management (EVM) system for IT investments is an example.

The Tevatron high energy particle collider facility at Fermilab, located at Batavia, Illinois, was one of the first large scientific user facilities when it was commissioned in early eighties. It remains the largest active facility for high-energy physicists until the commissioning of the Lepton Hadron Collider facility, currently being built at Geneva, Switzerland. However, the new facility will not 
be fully useable to scientists until the year 2009. In the mean time, the scientific community desperately needs much higher performance from the existing Tevatron. To meet the increasing need for higher performance, Fermilab has undertaken various projects to improve existing systems associated with its accelerator complex. One of the larger projects is the upgrade of the Beam Position Monitor (BPM) system associated with Tevatron. The objective of the project was to replace the old BPM system that was originally installed during early 1980s. The conceptual design of the system began during June 2003. The duration of the formalized project Work Breakdown Structure (WBS) was from November 2003 to June 2005.

A phase based project management methodology based on EVM principles proved to be advantageous to the system development effort. Deploying a full-scale EVM system was not an objective for the project. However, the estimated project cost was large enough to justify the application of some of the EVM methodology to manage the project efficiently. Three specific benefits were realized using this approach were: a) the methodology provided the discipline of assessing the cost and schedule impacts early so that associated risks could be managed early and appropriately; b) EVM data collected throughout the project provided insights about the dynamics of system development processes in a typical scientific organization. The experience gained is already proving to be valuable for planning another similar project; c) experiences gained in the EVM analysis process might be useful for future projects where EVM system might be mandatory.

The planned system upgrade was significantly more that a legacy system upgrade as it included major enhancements in features of the system and new signal processing capabilities to increase the functionalities of the Tevatron. The new system is capable of acquiring and processing a very large amount of data rapidly from the complex beam path of the Tevatron.

The schedule for the installation and commissioning was tightly driven by the accessibility to the Tevatron accelerator service buildings and general operation of the facility. When the accelerator was in operation, engineers could take crucial test data to develop algorithms. However, any installation could only be done during temporary shutdowns of the accelerator. On the other hand, during accelerator shutdown, no test data could be taken to validate the system. The commissioning of the system took longer than expected, since it involved an iterative process of gathering data using new BPMs installed in phases, understanding the errors, debugging the software and firmware, and finally, validating the system by collecting and studying new data. In this paper, an attempt has been made to understand project characteristics using EVM measures.

\section{BACKGROUND}

The BPM system consists of 236 beam position monitor channels around the underground tunnel of the Tevatron accelerator. Above ground control systems are attached to these monitors using pickup cables. When the Tevatron collider is operational, signals received from the BPMs are used to perform a number of control and diagnostic tasks. The old system could only capture the proton signals. The new system is able to capture combined proton and antiproton signals and separate antiproton signals from the combined signal. This significant enhancement was beyond the range of technical capabilities, both hardware and software, when the Tevatron was constructed in early eighties. The new system takes advantage of exceptional progresses made in the hardware and software technologies in past two decades.

As shown in the Fig. 1, the system has four major subsystems. Electronics subsystem includes $27 \mathrm{VME}$ based hardware units consisting of a timing module, multiple filter modules and multiple special purpose Digital Receiver boards purchased from Echotek Corporation. The programmable timing module to handle sensitive various clock events was developed in-house. Each Echotek board is paired with a filter board fitted with high-end filters. Each hardware unit resides in each of the 27 service buildings around the accelerator. Hardware units are integrated with the accelerator with the front-end software. The front-end software is primarily a VxWorks based data acquisition system. It performs preliminary acquisition and processing of data and communicates to the main accelerator control system.
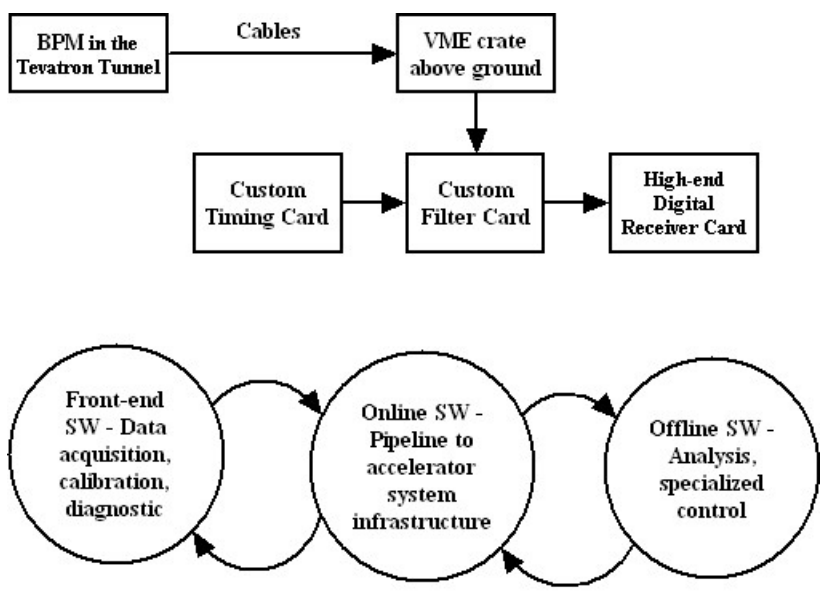

Fig. 1: BPM Subsystems

This subsystem, developed using modern methodologies, would remain easily extendable, maintainable, and possibly reusable, in other monitoring system projects. The online accelerator control software system provides accelerator operator interfaces, keeps track of the accelerator communication, system parameters and data. The online software component of the project is mostly an enhanced version of the existing software. The new development included modifications to software libraries and user interfaces. The offline software subsystem 
required significant amount of work, particularly in the areas of understanding the nature of the signals and necessary algorithms for signal processing, calibration and diagnostics.

\section{Project Organization}

At Fermilab, projects are generally organized using traditional work package model, where most activities span the entire duration of the project. However, to account for the significant software development effort, a phase-based approach was used to design the project WBS. Following system and project characteristics were important considerations during its design.

\section{System characteristics}

- The system is one of a kind system. Once built and commissioned, it would not be exactly duplicated anywhere else

- Desired system capabilities, although envisioned accurately in requirements document, could not be quantified fully before commissioning

- $\quad 27$ crates had to be installed and commissioned 27 service buildings handling a "live" operating accelerator. In some cases, old electronics had to be decommissioned simultaneously to install new electronics.

Project characteristics:

- Hardware and software co-design: The software development and hardware prototyping occurred simultaneously.

- "Lock-step" system installation and commissioning: The new BPM system was installed and commissioned around the accelerator in predetermined phases.

- System validation: During the commissioning phase, significant amount of effort was spent studying the data provided by the newly installed BPM electronics. Then, the data collected in phases was used to validate the full system incrementally.

As shown in the Fig. 2, the project WBS is composed of five major sections. Four large rectangles represent four major system life-cycle phases, namely design, fabrication, installation, and commissioning. The project management component, covering the work for all phases, includes project management support and technical coordination activities. The baseline estimate for the project was done using proven techniques of project management, including interviews with subject matter experts and detailed work and material estimation. MS project 2002 project management tool, with custom fields and macros, was used to organize and monitor the project. The data associated with the project earned value measures was collected for each system life cycle phases for each subsystem. This particular WBS designed allowed us to isolate the statistics on system components into well-defined system life-cycle phases.

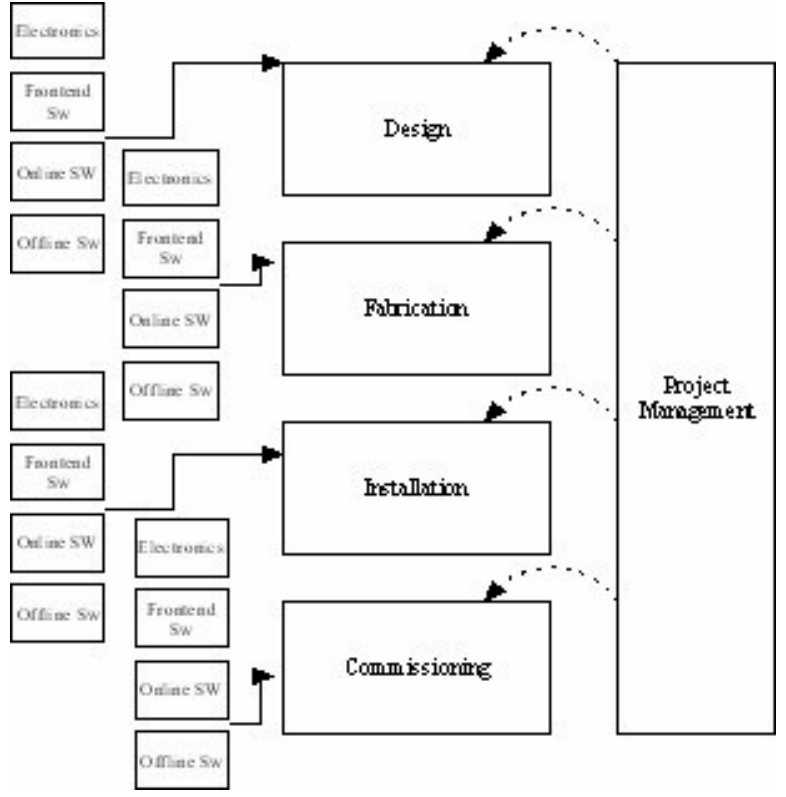

Fig. 2: Design of the phase-based WBS

\section{EVM DATA ANALYSIS}

Following the phase based WBS described above various earned value management data were collected throughout the project. During the project life cycle, there were several adjustments to the project WBS due to both external and internal changes that affected the project cost and schedule, As a result, total estimated cost of the project changed. To understand this variance, the total estimated cost at completion (TEC) for each phase of the project was calculated. One of the two EVM measures used in this paper is the earned value measure, also called Budgeted Cost of Work Performed (BCWP). BCWP is calculated from the value of the work performed up to a particular WBS status date. This measure is important to assess long term performance of the WBS. The other measure is the Estimate at Completion (EAC). The EAC measure for a particular task is defined to be the sum of the cost actually incurred up to the WBS status date and the cost estimated for remaining work to be done for that task. EAC proved to be an excellent measure for analyzing the performance during a particular life cycle phase. For the BPM project, TEC, BCWP, and EAC were calculated at the monthly status date for the WBS. Instead of TEC and BCWP by themselves, the variance of the BCWP from TEC provided more insight into the evolution of the project.

Since profiles for EVM measures remained steady for the first three months of the project, the data from this period is generally ignored in this paper

Comparing with the history of project activities, it is clear that the variance of the BCWP from TEC characterizes the general behavior of the project as it evolves through its life cycle. In Fig. 3, the cost variance for the total project, fabrication, and project management are shown. The variance for the total project dropped after the major electronic purchases were finalized in August of 2004. 


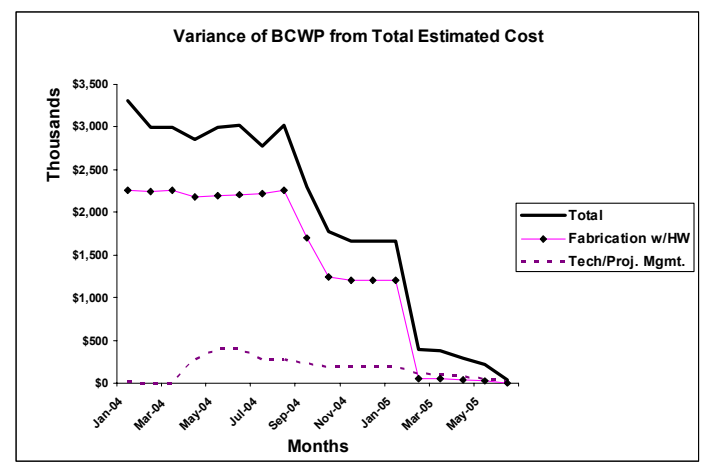

Fig. 3: Project EVM view

However, software fabrication work ramped up only after system design was reasonably understood. Project management cost also included technical coordination efforts. Although the project management work began early in the project, the technical coordination effort increased significantly during and after the month of February 2004. The variance remained small throughout the rest of the project. Isolating the almost steady level of work for project and technical management out of actual development work was useful because it allowed for examination of the development work by itself.

Design, installation, and commissioning activities are compared in Fig. 4. During the period of May 2004 to September 2004, unexpected variations in the graph characterizes the preparation period for the shutdown of the accelerator in September 2004. This chart clearly indicates reshuffling of priorities associated with the external constraint. During this period, a significant progress was made by installing and commissioning the first crate in the accelerator to collect early data. Without this extra effort, the system design could be validated early using the early data collected before the accelerator shut down.

For a scientific project, the design phase seems to be the most critical portion of the project. During the design phase of this project, the investigation for possible technical choices involved careful analysis of proton data collected using an older version of the Digital Receiver board, generic filters and operational features of the Tevatron under full-scale operation. At the same time, new algorithms were developed using the analysis of real data and simulations. The design was presented to a group of accelerator experts during a Technical Choice Review. However the design process for the project continued to evolve as designers discovered technical caveats and complexities of the proposed system.

Although the basic requirements for the system needed a significant amount of understanding, it was fairly stable at the beginning of the project. However, it was clear that the design process must accommodate a continuous process of discovery. It was clear that the variation in the project cost due to the influence of continuous discovery must be taken into account during the initial development of the cost estimates for scientific projects.

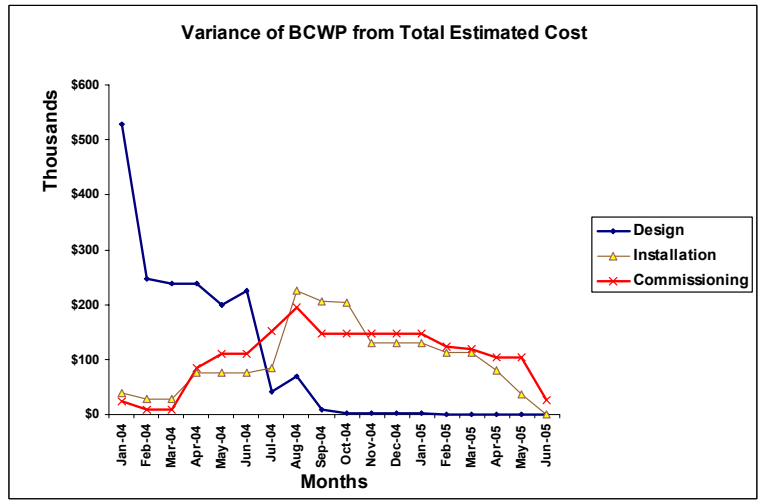

Fig. 4: Measures of changes in priorities

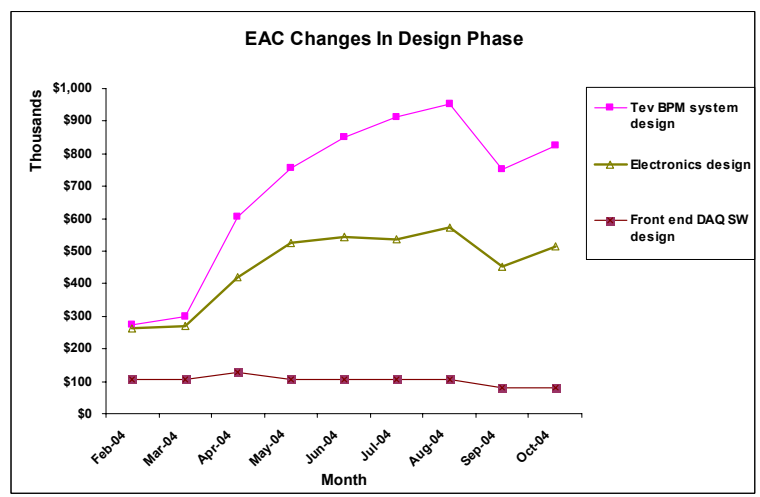

Fig. 5: Influence of discovery on design

The EAC data gathered for the design phase was a revealing indicator of the dynamics of the system design process. Fig. 5 shows graphs of the EAC data collected from February 2004 to October 2004 spanning the critical period of the electronic design. It presents the total EAC for the project during design, EAC for electronic design and front-end software design.

As mentioned before, the design of the electronics also included the design of the firmware. Electronics design influenced the total design cost significantly while software design cost remained fairly steady. It should be noted that these values do not include any material purchases.

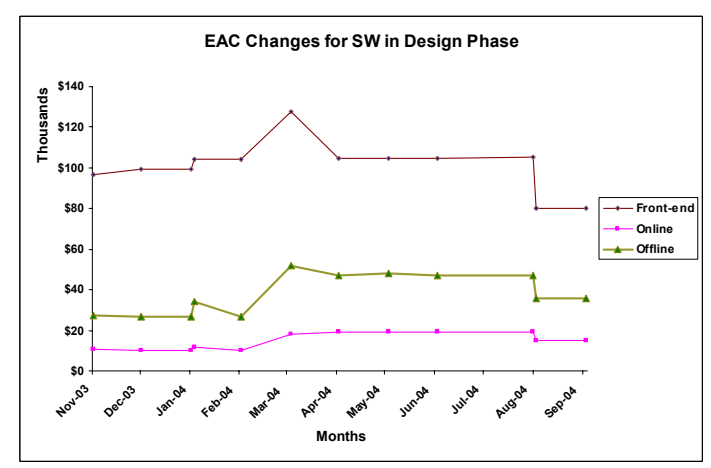

Fig. 6: Steady state software design 
The cost increases for the firmware component in the design phase was due to of larger than expected design effort for hardware enhancements. Cost increases in the Fig. 5 coincided with increased design change efforts. This excess cost can be attributed to the cost of discovery as well. Major enhancements to the new hardware system involved:

- The firmware in the timing modules was a significant design effort

- Enhanced digital receiver boards significantly modified by vendor. Cost of these boards is the most significant portion of total cost of the project. To minimize risks, designers used detailed cost/benefit analysis to decide on make or buy decisions, bidding process, prototype approval, and long production lead time. Significant amount of simulation work was also necessary

- Custom analog/anti-aliasing filters were purchased. Defining the filter specification with significantly tight tolerances was a significant effort. Finding an adequate vendor impacted the schedule.

In contrast with the electronic design, the EAC plot for the front end software indicates stability of the software design process during the design phase. The VxWorks based data acquisition software was well-understood by the team. Experts from other areas provided accurate interface information.

Using EAC variables, Fig. 6 presents the comparison of front end software with the online software and offline software. Like front end software, online software is wellunderstood. On the other hand, EAC values increases for the offline software. This component of the Tevatron software involved understanding completely new algorithms for the signal logic and the separation of proton/antiproton signals.

In the Fig. 7, the data on the variance of BCWP from TEC for software implementation indicates a general pattern of downward slope.

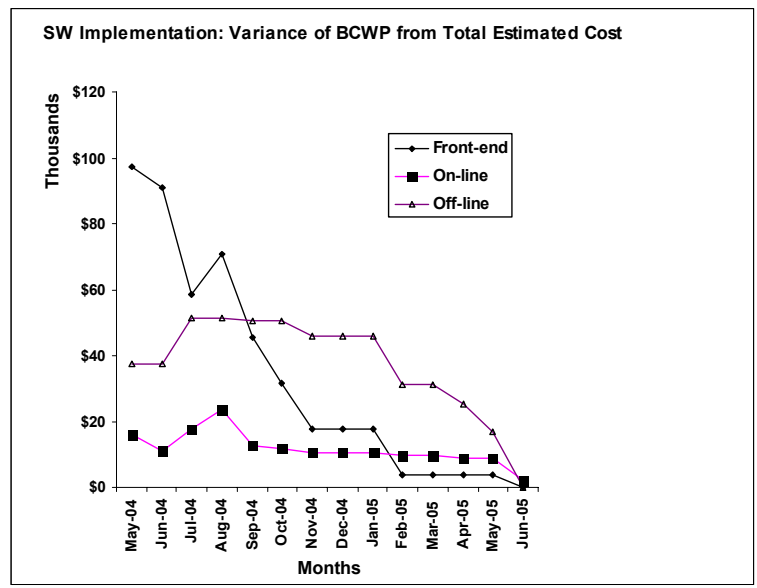

There were general increases in effort around July 2004 that could be attributed to early efforts on getting the first version of the software ready for the preliminary installation of the first BPM system before the Tevatron shutdown.

\section{CONCLUSION}

Many scientific projects are not large enough to justify the implementation of a full-fledged EVM system. However, if an infrastructure exists and it is possible to collect some of the EVM data easily, such measures may prove to be powerful tools for planning and estimating similar projects in the future. In the Tevatron BPM project, EAC and the variance between TEC and BCWP provided important clue to the evolution of the hardware and software co-design process in a scientific environment. Both of these measures depend on the project baselines and status of the project at equal intervals. Since tasks associated with phases and subsystems in the WBS were isolated from each other, it was only necessary to wait for the end of each phase of a subsystem to obtain a good understanding of the behavior of the development process for the phase and subsystem. This knowledge could be used effectively for future projects in Fermilab. For example, since types of resources needed for each life cycle phase are different, such quantification could be used to plan staff allocations for organizations involved with multiple projects. This information is also valuable for long-term budgeting and proposal processes.

Another important lesson learned from this exercise is that cost and schedule for a complex hardware/software system development is highly sensitive to the environment it resides in. The early quantification of the degree of scientific discoveries required during later phases of the project is essential for completing a project within its cost and schedule thresholds. When matched to the similar scientific environment, historical profiles of phase based measures are expected to behave similarly.

\section{ACKNOWLEGEMENT}

The author wishes to acknowledge the support of her supervisor Dr. Stephen Wolbers and Fermi National Accelerator Laboratory, operated by Universities Research Association Inc. under Contract No. DE-AC02$76 \mathrm{CH} 03000$ with the United States Department of Energy.

\section{REFERENCES}

[1] Report on the Project Management Panel of The University of California President's council on the National Laboratories, February 17-18, 2000

[2] EIA Standard EIA-748-A, "Earned Value Management Systems", January 2002

[3] B. Banerjee, "Managing Discovery Risks - A Tevatron Case Study," Proceedings of $5^{\text {th }}$ Annual UT/IEEE Engineering Management Conference, September, 2004 